\title{
HEAVY QUARK SYMMETRIES AND HEAVY MESON MOLECULES*
}

\author{
FENG-KUN GUO \\ Helmholtz-Institut für Strahlen- und Kernphysik and Bethe Center for Theoretical Physics, \\ Universität Bonn, D-53115 Bonn, Germany \\ CARLOS HIDALGO-DUQUE \\ Instituto de Física Corpuscular (IFIC), Centro Mixto CSIC-Universidad de Valencia, \\ Institutos de Investigación de Paterna, Aptd. 22085, E-46071 Valencia, Spain \\ JUAN NIEVES \\ Instituto de Física Corpuscular (IFIC), Centro Mixto CSIC-Universidad de Valencia, \\ Institutos de Investigación de Paterna, Aptd. 22085, E-46071 Valencia, Spain \\ MANUEL PAVÓN VALDERRAMA ${ }^{\dagger}$ \\ Institut de Physique Nucléaire, Université Paris-Sud, \\ IN2P3/CNRS, F-91406 Orsay Cedex, France \\ pavonvalderrama@ipno.in2p3.fr
}

Published 14 January 2014

\begin{abstract}
A few of the newly discovered XYZ states might be bound states of a heavy meson and antimeson. These molecular states, as they are also known, respect heavy quark symmetries at the potential level and as a consequence they might appear in multiplets. Here we exploit their symmetry to predict new states from already known ones. For instance, we expect the existence of a bottom counterpart of the $X(3872)$ at $10580 \mathrm{MeV}$. Conversely, the assumption that the $Z_{b}(10610)$ and $Z_{b}^{\prime}(10650)$ are molecular implies a pair of analogous resonances with hidden charm located at 3870 and $4010 \mathrm{MeV}$. They might be identified with the $Z_{c}(3900)$ and $Z_{c}(4025)$, supporting the idea that they are $1^{+-} D \bar{D}^{*}$ and $D^{*} \bar{D}^{*}$ bound states.
\end{abstract}

Keywords: Meson-meson interactions; heavy quarkonia; exotic mesons.

PACS numbers: 13.75.Lb, 14.40.Pq, 14.40.Rt

*This is an Open Access article published by World Scientific Publishing Company. It is distributed under the terms of the Creative Commons Attribution 3.0 (CC-BY) License. Further distribution of this work is permitted, provided the original work is properly cited.

${ }^{\dagger}$ Speaker. 


\section{Introduction}

The experimental discovery of the XYZ states, a series of hidden charm (bottom) resonances that do not fit into the canonical charmonium (bottomonium) spectrum, has opened new vistas on hadron spectroscopy. ${ }^{1}$ Probably a considerable fraction of these states are exotics, that is, they are not quark-antiquark states and require nonstandard explanations, for instance tetra-quarks, hadrocharmonia (a quarkonium core surrounded by a light quark-antiquark cloud), hybrids and molecules. Here we are concerned with the latter, hadronic molecules, bound states of heavy hadrons theorized thirty years ago. ${ }^{2}$ The molecular hypothesis is particularly enticing for XYZ states that are located in the vicinity of an open heavy flavor threshold. The most striking example is the $X(3872),{ }^{3}$ which within experimental uncertainty overlaps with the $D^{0} \bar{D}^{0 *}$ threshold, ${ }^{4}$ motivating the idea that the $X(3872)$ is a bound state of this meson-antimeson pair. ${ }^{5}$ Yet, this is not the only XYZ state that has been proposed to be a molecule. Other well-known examples are the $Z_{b}(10610)$ and $Z_{b}(10650)$ resonances discovered by Belle, ${ }^{6-8}$ which are thought to be $B \bar{B}^{*}$ and $B^{*} \bar{B}^{*}$ states. $^{9,10}$

Heavy meson-antimeson molecules are very interesting objects from the theoretical point of view. The reason is that they are highly symmetric: owing to their heavy quark content they are subjected to heavy quark symmetries, ${ }^{11,12}$ while their light quark content implies that they also obey isospin and $\mathrm{SU}(3)$ flavor symmetry and that their low energy interactions with pions are constrained by chiral symmetry. That is, we can predict the existence of so far unobserved molecular states from the ones that have been already discovered. However, the prediction requires knowledge of the binding mechanism. The previous symmetries do not manifest directly in the molecular spectrum, but only indirectly: what they constrain is the dynamics of the mesons conforming the bound states. As a consequence we face a serious problem: predictions will be different depending on the details of the potential binding the meson and antimeson, particularly at short distances. Yet there is a way out. Effective field theories (EFTs) are systematic descriptions of low energy phenomena, which are based on renormalization group invariance, a principle that states that low energy physics do not depend on the explicit short distance details. Within EFTs the inherent ambiguity of the short range dynamics of mesons is translated into a theoretical uncertainty in the predictions that can be estimated. In the following lines of this contribution we will show how to use EFTs to make reliable predictions (with their corresponding theoretical errors) of new molecular states.

\section{Predicting New Molecular States}

A property of EFTs is that they are low energy expansions: we can distinguish a characteristic low energy scale $Q$, which corresponds to the energy range we want to describe, and a hard scale $M$, at which the theory now longer works. If we are considering a heavy meson-antimeson pair, $Q$ can be identified with the pion mass or the average momentum of the mesons within the molecule, while $M$ represents 
the rho mass or the natural momentum scale at which the hadrons are no longer point-like (i.e. around $0.5-1 \mathrm{GeV}$ ). Then EFT predictions take the form of a power series in terms of the small parameter $x_{0}=Q / M: A=\sum_{\nu=0}^{\nu_{\max }} A^{(\nu)}+\mathcal{O}\left(x_{0}^{\nu_{\max }+1}\right)$, where the theoretical error comes from truncation of the EFT series. The ordering of EFT operators within the power series goes under the name of power counting. Other characteristic of EFTs is that they require the inclusion of an ultraviolet cutoff $\Lambda$ as a device to guarantee that results do not depend on unknown short-range physics. If the power counting is well-formulated, calculations will be independent of the cut-off $\Lambda$ modulo the truncation error. EFT practice indicates that the optimal cut-off choice is $\Lambda \sim M$. Even though there is formal cut-off independence, harder cut-offs lead to subtle problems like loss of causality ${ }^{13}$ and breakdown of low energy theorems. ${ }^{14}$ Finally, a possible way to determine the uncertainty of EFT predictions is to vary the cut-off in a sensible range around $\Lambda \sim M$.

The EFT for heavy meson-antimeson molecules is really simple: at lowest or leading order (LO) the heavy meson-antimeson interaction consists solely of contact interactions, with pion exchanges entering at subleading orders. ${ }^{15,16}$ If we consider the heavy mesons $P, P^{*}=D, D^{*}\left(B, B^{*}\right)$, heavy quark spin symmetry (HQSS) reduces the number of contact interactions to two. ${ }^{17,16}$ There are interesting relations, for instance the potential in the $J^{P C}=1^{++}$and $2^{++}$channels is identical

$$
V_{\mathrm{EFT}}^{\mathrm{LO}}\left(1^{++}, D \bar{D}^{*}\right)=V_{\mathrm{EFT}}^{\mathrm{LO}}\left(2^{++}, D^{*} \bar{D}^{*}\right),
$$

which means that if the $X(3872)$ is indeed a $J^{P C}=1^{++} D \bar{D}^{*}$ bound state, we should expect a $2^{++}$counterpart, where explicit calculations locate it at $4012 \mathrm{MeV}^{18}$ (though the state could be rather broad owing to decay into $D \bar{D}$ via one pion exchange). Other relation is

$$
V_{\mathrm{EFT}}^{\mathrm{LO}}\left(1^{+-}, D \bar{D}^{*}\right)=V_{\mathrm{EFT}}^{\mathrm{LO}}\left(1^{+-}, D^{*} \bar{D}^{*}\right),
$$

which explains the locations of the $Z_{b}(10610)$ and $Z_{b}^{\prime}(10650)$ (and also of the recent $Z_{c}(3900)^{19,20}$ and $\left.Z_{c}^{\prime}(4025)^{21}\right)$ with respect to their thresholds. Apart from HQSS, heavy flavor symmetry (HFS) is also an important symmetry for molecular states. According to HFS, the potential among heavy mesons does not depend on the flavor of the heavy quark. For instance we have

$$
V_{\mathrm{EFT}}^{\mathrm{LO}}\left(1^{+-}, D \bar{D}^{*}\right)=V_{\mathrm{EFT}}^{\mathrm{LO}}\left(1^{+-}, B \bar{B}^{*}\right)
$$

that is, if there is a $Z_{b}\left(Z_{b}^{\prime}\right)$ it should have a hidden charm partner, the $Z_{c}\left(Z_{c}^{\prime}\right)$. Explicit calculations locate the $Z_{c}\left(Z_{c}^{\prime}\right)$ at $3870 \mathrm{MeV}(4010 \mathrm{MeV}),{ }^{22}$ not far from the experimental $Z_{c}(3900)\left(Z_{c}^{\prime}(4025)\right)$. This is a strong hint that the recently discovered $Z_{c}(3900)$ and $Z_{c}^{\prime}(4025)$ resonances might be molecular and have the quantum numbers $1^{+-}$.

In Table 1 we review the list of molecular states that can be predicted from the $X(3872)$ and $Z_{b}(10610)$, indicated as input within the table, plus the assumption that they are molecular. For determining the errors we consider two possibilities: to vary the cut-off within a sensible range and to propagate the error in the potential 


\section{F.-K. Guo et al.}

Table 1. Predictions of isoscalar and isovector molecular states (in $\mathrm{MeV}$ ) and comparison with possible experimental candidates. "V" indicates a virtual (instead of bound) state. " $C_{0 X}$ " and " $C_{1 Z}$ " labels whether the EFT potential is that of the $X(3872)$ or the $Z_{b}$ 's.

\begin{tabular}{|c|c|c|c|c|c|}
\hline$V_{C}$ & $I\left(J^{P C}\right)$ & Mesons & $\mathrm{M}(\Lambda=0.5 \mathrm{GeV})$ & $\mathrm{M}(\Lambda=1 \mathrm{GeV})$ & Exp. \\
\hline \multirow[t]{5}{*}{$C_{0 X}$} & $0\left(1^{++}\right)$ & $\overline{D \bar{D}^{*}}$ & 3871.68 (input) & 3871.68 (input) & $3871.68 \pm 0.17^{4}$ \\
\hline & $0\left(2^{++}\right)$ & $D^{*} \bar{D}^{*}$ & $4012_{-5}^{+4}$ & $4012_{-12}^{+5}$ & $?$ \\
\hline & $0\left(1^{++}\right)$ & $B \bar{B}^{*}$ & $10580_{-8}^{+9}$ & $10539_{-27}^{+25}$ & ? \\
\hline & $0\left(2^{++}\right)$ & $B^{*} \bar{B}^{*}$ & $10626_{-9}^{+8}$ & $10584_{-27}^{+25}$ & $?$ \\
\hline & $0\left(2^{+}\right)$ & $D^{*} B^{*}$ & $7322_{-7}^{+6}$ & $7308_{-20}^{+16}$ & $?$ \\
\hline \multirow[t]{5}{*}{$C_{0 Z}$} & $1\left(1^{+-}\right)$ & $\overline{B \bar{B}^{*}}$ & $\begin{array}{c}10602.4 \pm 2.0 \\
\text { (input) }\end{array}$ & $\begin{array}{c}10602.4 \pm 2.0 \\
\text { (input) }\end{array}$ & $\begin{array}{c}10607.2 \pm 2.0^{6} \\
10597 \pm 9^{8}\end{array}$ \\
\hline & $1\left(1^{+-}\right)$ & $B^{*} \bar{B}^{*}$ & $10648.1 \pm 2.1$ & $10648.1_{-2.5}^{+2.1}$ & $\begin{array}{c}10652.2 \pm 1.5^{6} \\
10649 \pm 12^{8}\end{array}$ \\
\hline & $1\left(1^{+-}\right)$ & $D \bar{D}^{*}$ & $3871_{-12}^{+4}(\mathrm{~V})$ & $3837_{-35}^{+17}(\mathrm{~V})$ & $\begin{array}{l}3899.0 \pm 3.6 \pm 4.9^{19} \\
3894.5 \pm 6.6 \pm 4.5^{20}\end{array}$ \\
\hline & $1\left(1^{+-}\right)$ & $D^{*} \bar{D}^{*}$ & $4013_{-11}^{+4}(\mathrm{~V})$ & $3983_{-32}^{+17}(\mathrm{~V})$ & $4026.3 \pm 2.6 .^{21}$ \\
\hline & $1\left(1^{+}\right)$ & $D^{*} B^{*}$ & $7333.6_{-4.2}^{+0.1}(\mathrm{~V})$ & $7328_{-14}^{+5}(\mathrm{~V})$ & $?$ \\
\hline
\end{tabular}

to the results by means of an explicit calculation. For the standard EFT error - the $Q / M$ uncertainty in the expansion - we have chosen the first option, which is why in Table 1 we have included the $\Lambda=0.5 \mathrm{GeV}$ and $1 \mathrm{GeV}$ results (the error being the difference). In addition HQSS and HFS are not exact, but approximate, the error being $\Lambda_{\mathrm{QCD}} / m_{Q}$ in the potential, with $\Lambda_{Q C D} \sim 0.3 \mathrm{GeV}$ and $m_{Q}$ the heavy quark mass. Here we have propagated from the potential, with the errors indicated by the subscripts and superscripts in the numbers of Table 1. Apart from the prediction of theoretical candidates for the $Z_{c}(3900)$ and $Z_{c}^{\prime}(4025)$ resonances, we also predict the hidden bottom counterpart of the $X(3872)$, the $X_{b}(10580)$. A possible way to detect the $X_{b}$ in the future is by looking to the $\Upsilon(1 S, 2 S) 3 \pi$ invariant mass distribution. However, unlike the $X(3872)$ and the $J / \Psi 2 \pi$ channel, the $X_{b}$ is not expected to be detectable in the $\Upsilon(1 S, 2 S) 2 \pi$ channel, the reason being that the isospin breaking effects are probably tiny in the $X_{b}$.

To summarize, in this contribution we have analyzed the consequences of HQSS and HFS in the spectrum of heavy meson-antimeson molecules. For that we use the EFT framework, which allows us to make reliable estimations of the errors in the theoretical predictions (in contrast to phenomenological approaches, where errors are difficult to estimate). Apart from explaining the spectrum of a few hidden charm and bottom resonances, we can use HQSS and HFS to predict the existence of so far unobserved states. Regarding the recently discovered $Z_{c}(3900)$ and $Z_{c}(4025)$, our results give support to the hypothesis that they are indeed molecular and that their quantum numbers are $1^{+-}$, though further efforts are still required.

\section{Acknowledgments}

This work is supported in part by the DFG and the NSFC through funds provided to the Sino-German CRC 110 "Symmetries and the Emergence of Structure in QCD", by the NSFC (Grant No. 11165005), by the Spanish Ministerio de Economía y 
Competitividad and European FEDER funds under the contract FIS2011-28853C02-02 and the Spanish Consolider-Ingenio 2010 Programme CPAN (CSD200700042), by Generalitat Valenciana under contract PROMETEO/2009/0090 and by the EU HadronPhysics2 project, grant agreement no. 227431. C.H.-D. thanks the support of the JAE-CSIC Program. M.P.V thanks the organizers of the workshop for their financial assitance.

\section{References}

1. N. Brambilla et al., Eur. Phys. J. C 71, 1534 (2011), 1010.5827.

2. M. B. Voloshin and L. B. Okun, JETP Lett. 23 (1976) 333 [Pisma Zh. Eksp. Teor. Fiz. 23 (1976) 369].

3. Belle Collaboration, S. Choi et al., Phys. Rev. Lett. 91, 262001 (2003), hepex/0309032.

4. Particle Data Group, J. Beringer et al., Phys. Rev. D 86, 010001 (2012).

5. N. A. Tornqvist, Phys. Lett. B 590, 209 (2004), hep-ph/0402237.

6. A. Bondar et al. [Belle Collaboration], Phys. Rev. Lett. 108, 122001 (2012) [arXiv:1110.2251 [hep-ex]].

7. I. Adachi et al. [Belle Collaboration], arXiv:1207.4345 [hep-ex].

8. I. Adachi et al. [Belle Collaboration], arXiv:1209.6450 [hep-ex].

9. M. B. Voloshin, Phys. Rev. D 84, 031502 (2011) [arXiv:1105.5829 [hep-ph]].

10. M. Cleven, F. -K. Guo, C. Hanhart and U. -G. Meissner, Eur. Phys. J. A 47, 120 (2011) [arXiv:1107.0254 [hep-ph]].

11. M. Neubert, Phys. Rept. 245, 259 (1994), hep-ph/9306320.

12. A. V. Manohar and M. B. Wise, Heavy Quark Physics (Cambridge University Press, Cambridge, 2000).

13. D. R. Phillips and T. D. Cohen, Phys. Lett. B 390, 7 (1997) [nucl-th/9607048].

14. E. Epelbaum and J. Gegelia, Eur. Phys. J. A 41, 341 (2009) [arXiv:0906.3822 [nuclth]].

15. S. Fleming, M. Kusunoki, T. Mehen, and U. van Kolck, Phys. Rev. D 76, 034006 (2007), hep-ph/0703168.

16. M. Pavon Valderrama, Phys. Rev. D 85, 114037 (2012) [arXiv:1204.2400 [hep-ph]].

17. M. T. AlFiky, F. Gabbiani, and A. A. Petrov, Phys. Lett. B 640, 238 (2006), hep$\mathrm{ph} / 0506141$.

18. J. Nieves and M. Pavon Valderrama, Phys. Rev. D 86, 056004 (2012) [arXiv:1204.2790 [hep-ph]].

19. M. Ablikim et al. [BESIII Collaboration], Phys. Rev. Lett. 110, 252001 (2013) [arXiv:1303.5949 [hep-ex]].

20. Z. Q. Liu et al. [Belle Collaboration], Phys. Rev. Lett. 110, 252002 (2013) [arXiv:1304.0121 [hep-ex]].

21. M. Ablikim et al. [BESIII Collaboration], arXiv:1308.2760 [hep-ex].

22. F. -K. Guo, C. Hidalgo-Duque, J. Nieves and M. Pavon Valderrama, Phys. Rev. D 88, 054007 (2013) arXiv:1303.6608 [hep-ph]. 